\title{
Optimización de la eficiencia en la conducción para rutas predeterminadas
}

\author{
Roberto García ${ }^{1}$, Alejandro G. Tuero ${ }^{1}$, Laura Pozueco $^{1}$, Xabiel G. Pañeda $^{1}$, Victor Corcoba ${ }^{1}, J^{\prime}$ sé A. \\ Sanchez ${ }^{1}$, David Melendi ${ }^{1}$, Abel Rionda ${ }^{2}$. \\ ${ }^{1}$ Departamento de Informática, Universidad de Oviedo \\ ${ }^{2}$ ADN Mobile Solutions. Parque Tecnológico. Gijón, Asturias, España \\ \{garciaroberto, garciatalejandro, pozuecolaura, xabiel, corcobavictor, sanchezsjose, melendi\} @ uniovi.es, \\ abel.rionda@adnmobilesolutions.com
}

\begin{abstract}
Resumen- The transport sector is one of the main causes of the emission of pollutants to the environment. Among the different alternatives for reducing consumption and emission of harmful particles the most attractive for professional transport companies is the use of efficient driving techniques as it allows to take advantage of the existing fleet without the need to invest in new vehicles and technology . In this work we determine the optimal driving technique to minimize fuel consumption in a route. The results can be applied in training courses in efficient driving. For the calculations we use real data of a professional bus fleet. Then, we develop a consumption model and, using route optimization, we determine the driving technique that minimizes the consumption in the analyzed route. The results indicate that efficient driving has a very significant influence on fuel consumption. With optimum driving, reductions in consumption of up to 15 liters $/ 100 \mathrm{~km}(28 \%$ of reduction) could be achieved in the analyzed route. For the whole company, this is a significant reduction of consumption, considering the large distances covered by the professionals of the transport sector.
\end{abstract}

Palabras Clave- Conducción eficiente, optimización de rutas, transporte profesional

\section{INTRODUCCIÓN}

Las técnicas de formación en conducción eficiente en flotas de transporte profesional están recibiendo mucha atención en los últimos tiempos. El consumo de combustibles fósiles hace del sector del transporte uno de los principales causantes de la emisión de $\mathrm{CO} 2$ y otras partículas contaminantes al ambiente. Para mitigar esta situación se puede mejorar la tecnología de los vehículos o hacer un uso más eficiente de la ya existente mediante programas de mantenimiento $y$ técnicas de conducción eficiente.
En las flotas de transporte profesional la opción más atractiva, y económica, es la utilización de técnicas de conducción eficiente ya que permite aprovechar la flota de vehículos existente sin necesidad de invertir en nuevos vehículos y tecnología. Para lograr una disminución relevante en el consumo de combustible es necesario formar adecuadamente a los conductores. Éstos controlan la aceleración, frenado, velocidad, rpms, marcha engranada, posición del vehículo en la calzada, por lo que su acción es determinante para lograr mejoras notables en el consumo de combustible y en la eficiencia medioambiental.

Los programas de conducción eficiente orientados a la formación deben conseguir que el conductor sea capaz de acometer las acciones de conducción necesarias, adaptadas al vehículo y la vía, para realizar una conducción segura y con un menor consumo de combustible. La evaluación del programa de conducción eficiente se llevará a cabo mediante el rendimiento de los conductores que lo integran, bien mediante comparaciones simples de consumos de combustible o analizando las acciones de conducción. Como el consumo de combustible depende de muchos factores ajenos a la acción de conducción (temperatura, tráfico, carga, etc), la manera más justa de evaluar el rendimiento de un conductor es mediante el análisis de su conducción (aceleración, deceleración, conducción en inercia, ralentí).

Asimismo, para evaluar de forma objetiva el rendimiento del conductor es necesario registrar todos los eventos del vehículo mediante la lectura de parámetros recogidos de la ECU (Engine Control Unit) a través del bus CAN [1] y almacenados en una base de datos para su análisis posterior. 
El objetivo de este artículo es determinar la técnica óptima de conducción en una ruta y vehículo determinados para minimizar el consumo de combustible. Así, se podrán mejorar las estrategias de formación y evaluación de los profesionales al posibilitar la comparación de la acción de conducción desarrollada con el óptimo para esa ruta y vehículo.

Aplicando técnicas de regresión lineal se ha obtenido un modelo de consumo para el vehículo analizado. Con el modelo de consumo se ha diseñado un algoritmo de optimización de rutas que permite realizar el mismo trayecto en el mismo tiempo, pero minimizando el consumo de combustible. Finalmente, a partir de la ruta óptima se determina la acción de conducción que posibilita el menor consumo de combustible en el trayecto analizado. Los resultados indican que, en el trayecto de $11.1 \mathrm{~km}$, se obtienen ahorros medios de 15 1/100km (-28\% de consumo). Para el mes completo, solamente contabilizando las rutas realizadas por el vehículo analizado, se habrían ahorrado 1008 litros de combustible.

Para la realización del trabajo se dispone de los datos reales de 16 compañías del transporte en España y Marruecos, donde se han recogido los datos de 880 conductores. Para el estudio que se presenta se ha seleccionado una compañía de autobuses urbanos debido a que el alto consumo de combustible de estos vehículos y el elevado número de horas diarias de funcionamiento generan un gran impacto en las condiciones medioambientales de la ciudad en la que prestan servicio. La compañía de autobuses opera en una ciudad española de más de 250,000 habitantes. Se ha analizado la información de un autobús urbano no articulado en una línea de transporte en el mes de diciembre de 2015, procesando 1,048,576 muestras con 21 parámetros cada una de ellas.

En la sección II se comentan los trabajos relacionados. En la sección III se describe el sistema de recogida y análisis de la información que será utilizada para el modelo de regresión de la sección IV. El algoritmo de optimización de rutas se implementa en la sección V que se aplicará al caso de estudio de una ruta real en la sección VI. Finalmente, la sección VII muestra las conclusiones y líneas de trabajo futuras.

\section{TRABAJOS RELACIONADOS}

Actualmente existe un gran número de investigaciones relacionadas con la conducción eficiente y sus beneficios a la hora de reducir el consumo de combustible en flotas profesionales y, por consiguiente, disminuir las emisiones de $\mathrm{CO} 2$ al ambiente. Así, Rutty et al. [2] evaluaron el efecto de la formación en conducción eficiente, concluyendo que, gracias a la formación de los conductores en ecodriving, las emisiones medias de $\mathrm{CO} 2$ se redujeron en $1.7 \mathrm{~kg}$ por vehículo y día. De la misma forma, los autores en [3], a través de un programa de cursos de formación en Grecia, demostraron que se puede ahorrar hasta un $10 \%$ de combustible. La evaluación llevada a cabo por Strömberg and Karlson [4] sobre programas de formación en conducción eficiente en Suecia demostró que las reducciones de consumo alcanzaban el 6,8\% para una flota de autobuses. También, mediante técnicas de monitorización continua, Vagg et al. [5] consiguieron reducir el consumo el 7.6\%. Otros trabajos en la misma línea, como los de Ferreira et al. [6] para una flota de autobuses en Lisboa, muestran reducciones de consumo de 3 a $51 / 100 \mathrm{~km}$ aplicando formación en técnicas de conducción eficiente. En España, Rionda et al. [7] obtuvieron reducciones de consumo del $10 \%$ aplicando diferentes técnicas de formación a los conductores de una empresa de autobuses urbanos.

Todos los trabajos mencionados presentan ahorros significativos en el consumo de combustible, aplicando técnicas de eco-driving. Sin embargo, el punto de comparación es respecto al consumo antes de la formación. No existe ningún método que permita estimar el máximo ahorro posible ni comparar la técnica de conducción con la ideal. En nuestro trabajo se ha realizado una estimación de la técnica ideal de conducción que permitiría alcanzar el mismo destino en el mismo tiempo de servicio con el mínimo consumo de combustible. Estos resultados pueden aplicarse en los cursos de formación para mejorar las técnicas de ecodriving, además de proporcionar un límite inferior de comparación para el consumo de combustible en unas condiciones determinadas.

Para desarrollar el algoritmo de optimización de rutas es necesario elaborar un modelo de consumo basado en datos reales de funcionamiento del vehículo bajo estudio. En el trabajo de Delgado et al. [8] para una flota de cinco autobuses se demostró que la velocidad, la aceleración y la distancia entre paradas eran suficientes para predecir el consumo de combustible con una precisión razonable. Otros factores como tamaño del vehículo y gradiente de la vía también influyen en el consumo [9]. En el caso presentado en este artículo se va analizar el mismo vehículo en la misma ruta, por lo que el tipo de vehículo y vía se mantienen invariables a lo largo del estudio. Otros factores como la carga del vehículo, tráfico, condiciones meteorológicas o la temperatura también afectarían al consumo. Al no disponer de esta información, en la elaboración del modelo se suponen las mismas condiciones en todos los casos.

Respecto a la optimización del consumo de un vehículo basado en un perfil de velocidad, se han utilizado varios métodos en la literatura [10], [11], [12]. La mayoría de estudios están basados en optimizar la utilización de energía, aunque hay otros en los que el objetivo es minimizar el tiempo de la ruta [13]. En este trabajo hemos seguido la técnica de programación dinámica tomando como función de coste a minimizar el consumo de combustible. De forma similar a Mensing et al. [14], se ha optimizado el consumo en 
una ruta en la que el vehículo recorre la misma distancia con las mismas paradas en el mismo tiempo, con un menor consumo de combustible. Otra aportación respecto a otros artículos es que para estimar y minimizar la función de coste se han utilizado los datos reales de consumo de un autobús urbano capturados a través de la ECU del vehículo.

\section{SISTEMA DE RECOGIDA Y ANÁLISIS DE LA INFORMACIÓN}

Para la captura de datos de los vehículos, almacenamiento y análisis se ha utilizado el dispositivo CATED BOX $^{1}$, desarrollado por la compañía ADN Mobile [15], [16], [17]. La arquitectura general de CATED BOX tiene dos subsistemas con diferentes componentes hardware y software: un sistema empotrado y el sistema central.

Un sistema empotrado lee la información del vehículo a través de la ECU (Engine Control Unit) y la envía a un sistema central para ser almacenada y procesada, como se indica en la Fig. 1. El sistema captura una serie de variables de la ECU que dependen de las características particulares del vehículo, entre las que se incluyen velocidad, revoluciones por minuto, aceleración longitudinal, ditancia total, consumo instantáneo, carga motor, peso total, marcha engranada, etc. Esta información recogida de la ECU se complementa con los datos de posición GPS y del acelerómetro.

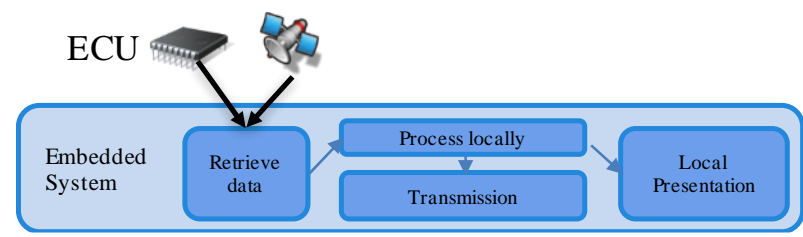

Fig. 1. Sistema empotrado de CATED BOX

El sistema central se encarga de recibir la información del sistema empotrado en los vehículos y almacenarla para su posterior análisis. Como se indica en la Fig. 2, el núcleo del sistema está compuesto por dos bases de datos. Una base de datos no relacional (CouchDB) para almacenar la información proveniente del sistema embarcado y una base de datos relacional (PostgreSQL) donde se almacenan los resúmenes de ruta y otra información complementaria. De forma periódica, se ejecutan varias tareas para leer datos de la base CouchDB, realizar operaciones y almacenar los resultados en la base de datos PostgreSQL. También se incluyen otros detalles proporcionados por las compañías de transporte, como información de vehículos, conductores y las rutas que realizan. Toda

\footnotetext{
${ }^{1}$ http://www.adnmobilesolutions.com/en/catedbox.html
}

esta información complementaria permite correlar la información del rendimiento del vehículo con un conductor o ruta particulares, como en el caso del trabajo que se presenta en este artículo.

El sistema central también incluye una serie de aplicaciones diseñadas para los diferentes perfiles de usuario, como pueden ser:

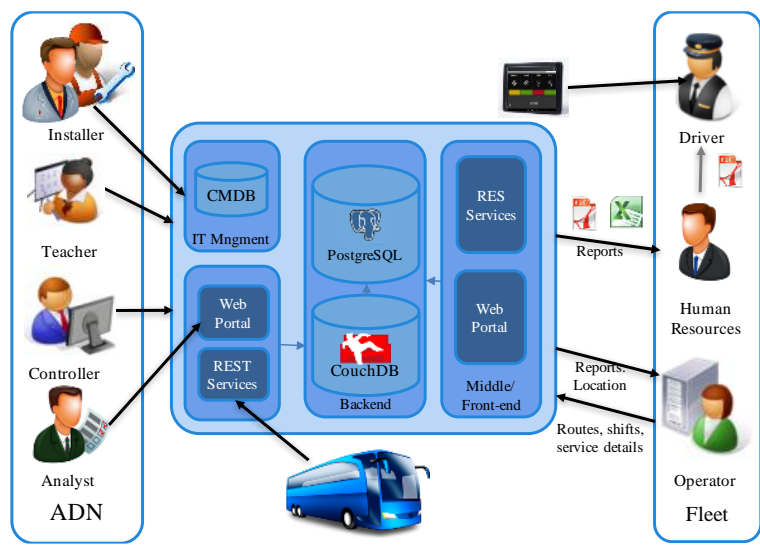

Fig. 2. Sistema central de CATED BOX

- Gestión y control de los dispositivos embarcados e infraestructura

- Generación de informes e indicadores de rendimiento para las compañías de transporte

- Generación de informes para proporcionar soporte a los formadores en conducción eficiente

Debido a las funcionalidades de la herramienta, CATED BOX puede utilizarse en las compañías de transporte para formar y evaluar el rendimiento de los conductores en lo referencte a eficiencia en la conducción. Los estudios realizados en este artículo están enfocados a proporcionar un soporte para la formación en conducción eficiente al indicar cuál sería la acción de conducción óptima par realizar un trayecto, minimizando el consumo de combustible y, por tanto, la emisión de partículas contaminantes al medioambiente. También sería de ayuda en los procesos de evaluación, ya que proporciona un umbral con el que comparar la eficiencia de cada ruta y vehículo.

\section{MODELO DE REGRESIÓN PARA EL CÁLCULO DEL CONSUMO DE COMBUSTIBLE}

Como paso previo a la construcción del algoritmo que optimiza el consumo en la ruta analizada se va a definir el modelo que permite determinar el consumo en función de la velocidad y la aceleración. Se ha analizado la información de un autobús urbano no articulado en una línea de transporte, procesando 1,048,576 muestras con 21 parámetros.

El análisis comienza con la recogida de datos de la ECU del vehículo mediante un dispositivo on-board diseñado a tal efecto, que se complementa con los datos 
de posición GPS y del acelerómetro. Con toda esta información se compone una traza que está formada por las variables que contienen toda la información sobre el rendimiento del vehículo, recogidas con una periodicidad de 1.5 segundos. El volumen de datos generado es de más de un millón de trazas.

Estos datos sufren un primer procesamiento para obtener los parámetros de cada una de las rutas. A continuación, se hace un filtrado de la información, con el objetivo de identificar aquellas trazas y rutas que no deben entrar a formar parte del modelo por presentar datos anómalos. Así, se eliminan del modelo las rutas y trazas que presenten valores no válidos como consumos instantáneos negativos, rpm fuera del rango de operación del vehículo, velocidades y marchas engranadas anormales, etc.

Para el algoritmo de optimización de la ruta es necesario determinar el consumo instantáneo de combustible en función de la velocidad actual, la velocidad anterior y la aceleración.

$$
\text { Consumo }_{\mathrm{k}}=\mathrm{f}\left(\mathrm{v}_{\mathrm{k}}, \mathrm{v}_{\mathrm{k}-1}, \mathrm{a}_{\mathrm{k}}\right)
$$

Antes de comenzar con la especificación del modelo hay que identificar si existe multicolinealidad, lo que indica relación de dependencia entre las variables predictoras. Cuando hay relación entre las variables predictoras algunas de ellas tienen que ser eliminadas para simplificar el modelo y mejorar su explicación. Una forma de medir la multicolinealidad es por medio de la matriz de correlación. Sin embargo, la multicolinealidad también puede aparecer cuando la variación de una variable predictora se explica por una combinación lineal de otros predictores. En este caso, Variance Inflation Factor (VIF) es el test de multicolinealidad recomendado para detectar relaciones significativas entre las variables independientes. Como indican los autores en [18], los problemas de multicolinealidad aparecen si VIF $>4$. Se ha calculado el factor VIF para las variables predictoras y no se ha detectado multicolinealidad.

Se han probado diferentes modelos de regresión, llegando a la conclusión que el modelo que mejor representa el consumo de combustible es el siguiente:

Consumo $_{\mathrm{k}}=\beta_{0}+\beta_{1} \cdot \mathrm{v}_{\mathrm{k}}+\beta_{2} \cdot \mathrm{v}_{\mathrm{k}-1}+\beta_{3} \cdot \mathrm{a}_{\mathrm{k}}+\beta_{4} \cdot\left(\mathrm{v}_{\mathrm{k}}-\mathrm{v}_{\mathrm{k}-1}\right)+$ $\beta_{5} \cdot \mathrm{v}_{\mathrm{k}}^{2}+\beta_{6} \cdot \mathrm{v}_{\mathrm{k}-1}^{2}+\beta_{7} \cdot \mathrm{a}_{\mathrm{k}}^{2}+\beta_{8} \cdot\left(\mathrm{v}_{\mathrm{k}}-\mathrm{v}_{\mathrm{k}-1}\right)^{2}$

Se ha utilizado el toolbox estadístico de MATLAB ${ }^{2}$ para implementar las regresiones. El modelo original utiliza 1,039,762 observaciones que se corresponden con el número total de trazas después del proceso de

${ }^{2}$ The Mathworks, Inc. Matlab R2015b: Statistics and Machine Learning Toolbox filtrado. La salida del modelo contiene el coeficiente constante $\beta_{0}$ y los coeficientes de las variables predictoras. Los coeficientes se estiman utilizando MLE (Maximum Likelihood Estimation) para minimizar el error cuadrático medio entre el vector de predicción $\hat{y}=\beta \cdot f(X)$ y la respuesta real, $\hat{y}-y$. El error RMS del modelo es 1.87.

Los términos del modelo de regresión se indican en la Tabla I. La primera columna indica el término incluido en el modelo, de acuerdo a la ecuación (2). La segunda columna es el valor del coeficiente $(\beta \mathrm{i})$, con el error estándar (SE) en la siguiente. La columna pValor es uno de los términos más importantes ya que representa el p-valor del estadístico $\mathrm{F}$ para el test de hipótesis. En todos los casos es menor que 0.01, indicando que todos los términos considerados son significativos en el modelo, para un nivel de significancia del $1 \%$.

Una vez eliminados los outliers, el modelo se ha ajustado utilizando 763,532 observaciones. Una medida de la bondad de ajuste del modelo es el coeficiente de determinación $\left(\mathrm{R}^{2}\right)$. Este estadístico indica el nivel de ajuste del modelo a los datos reales a predecir. En este caso, el coeficiente de determinación es de 0.972, próximo a la unidad, lo que sugiere que el modelo explica el $97.2 \%$ de la variabilidad de la variable dependiente.

Concluyendo, a partir de los resultados de los diferentes estadísticos puede afirmarse que el modelo representa con exactitud la variabilidad en el consumo de combustible en función de la velocidad y la aceleración consideradas.

Tabla I

COEFICIENTES DEL MODELO DE REGRESIÓN

\begin{tabular}{cccc}
\hline \hline Coeficiente & Estimación & SE & pValor \\
\hline$\beta_{0}$ & 2.495 & 0.0029 & $<0.01$ \\
$\beta_{1}$ & -1.065 & 0.0027 & $<0.01$ \\
$\beta_{2}$ & 1.356 & 0.0028 & $<0.01$ \\
$\beta_{3}$ & 0.261 & 0.0084 & $<0.01$ \\
$\beta_{4}$ & 0.000 & 0.0000 & NaN \\
$\beta_{5}$ & -0.094 & 5.5309 & $<0.01$ \\
$\beta_{6}$ & 0.094 & 5.4540 & $<0.01$ \\
$\beta_{7}$ & 1.136 & 0.0066 & $<0.01$ \\
$\beta_{8}$ & 0.537 & 0.0004 & $<0.01$ \\
\hline \hline
\end{tabular}

V. ALGORITMO DE OPTIMIZACIÓN DE RUTAS

Para optimizar el consumo de una ruta se ha diseñado un algoritmo para cada ciclo de eco-driving. Cada uno de estos ciclos comienza y finaliza en una situación de velocidad cero. De esta forma, una ruta está compuesta por la concatenación de varios ciclos de eco-driving. El objetivo del algoritmo es minimizar la función de coste consumo de combustible en cada ciclo, optimizando el perfil de velocidad y aceleración.

El sistema se rige por las siguientes ecuaciones, considerando la distancia recorrida (x), la velocidad (v), aceleración (a) y tiempo (t):

$$
x_{k+1}=x_{k}+v_{k} \cdot \Delta t+\frac{1}{2} \cdot a_{k} \cdot \Delta t^{2}
$$




$$
v_{k+1}=v_{k}+a_{k} \cdot \Delta t
$$

Se va a utilizar una aproximación tridimensional [14] para conseguir realizar el mismo recorrido en el mismo tiempo minimizando el consumo, de acuerdo con el principio de optimización de Bellman [19]. Así, en el algoritmo cada uno de los estados (X) considerados está compuesto de tres variables, con los estados inicial $\mathrm{X}_{0} \mathrm{y}$ final $\mathrm{X}_{\mathrm{f}}$ indicados en la Ec. 5:

$$
X=\left[\begin{array}{l}
t \\
x \\
v
\end{array}\right], X_{0}=\left[\begin{array}{l}
0 \\
0 \\
0
\end{array}\right], X_{f}=\left[\begin{array}{c}
t_{f} \\
x_{f} \\
0
\end{array}\right]
$$

Para implementar el algoritmo se sigue el esquema indicado en la Fig. 3, donde se muestran el tiempo final, la distancia final y la limitación de velocidad máxima para ese tramo.

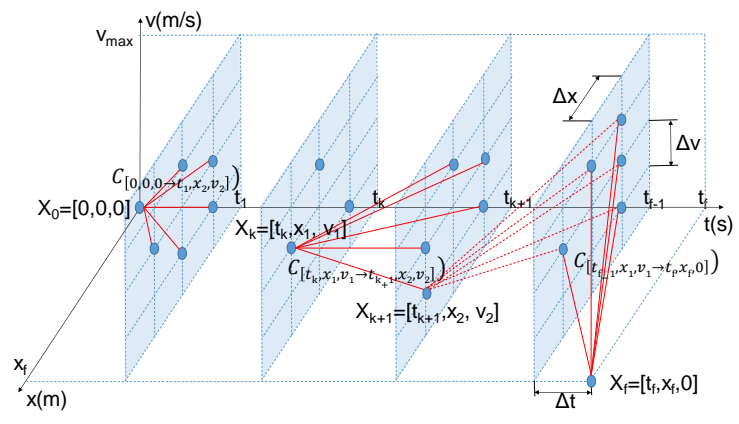

Fig. 3. Cálculo de costes en el método tridimensional

En primer lugar, se recorre el trayecto en sentido inverso, partiendo del estado final $\mathrm{X}_{\mathrm{f}}$ hasta llegar al estado inicial $\mathrm{X}_{0}$, analizando todos los posibles estados en el gráfico tridimensional y almacenando el índice que minimiza el coste energético. Una vez conocida la trayectoria a seguir se recorre nuevamente el trayecto desde el estado inicial al final siguiendo el camino de mínimo consumo y calculando los perfiles de velocidad, aceleración y consumo obtenidos.

Partiendo del estado final, en cada iteración del algoritmo se calcula la función de coste (consumo, de acuerdo a las Ec. 1,2) en base a las velocidades $v_{2}, v_{1} y$ la aceleración $\mathrm{a}_{1}$. Se analizan todas las opciones posibles $\left[\mathrm{t}_{\mathrm{k}+1}, \mathrm{x}_{2}, \mathrm{v}_{2}\right]$ para alcanzar el estado $\left[\mathrm{t}_{\mathrm{k}}, \mathrm{x}_{1}, \mathrm{~V}_{1}\right] \mathrm{y}$ se almacena el índice que minimiza la función de coste, de acuerdo con la Ec. 6.

$$
C_{\left[t_{k}, x_{1}, v_{1}\right]}=\min _{x 2, v 2}\left(C_{\left[t_{k+1}, x_{2}, v_{2}\right]}+C_{\left[t_{k}, x_{1}, v_{1} \rightarrow t_{k+1}, x_{2}, v_{2}\right]}\right)
$$

Se indican a continuación los resultados de la ejecución del algoritmo para un tramo ejemplo de 200m, en un tiempo de 30s, con límite de velocidad máxima de $10 \mathrm{~m} / \mathrm{s}$ y límite de aceleración de $\pm 3 \mathrm{~m} / \mathrm{s}^{2}$. En la Fig.4 se muestra una representación tridimensional la evolución de las variables tiempo, distancia y velocidad. En las siguientes figuras puede apreciarse cómo para recorrer el trayecto con el menor consumo posible se incrementa progresivamente la velocidad con una aceleración suave, que no supere los límites establecidos para el confort y seguridad de los pasajeros, hasta llegar a una zona en la que la velocidad se mantiene constante. Posteriormente, se aprovecha la inercia del vehículo, para obtener un consumo nulo hasta la detención. En el tramo analizado, el consumo total ha sido de 0.0862 litros, con una media de 43.133 litros/100km.

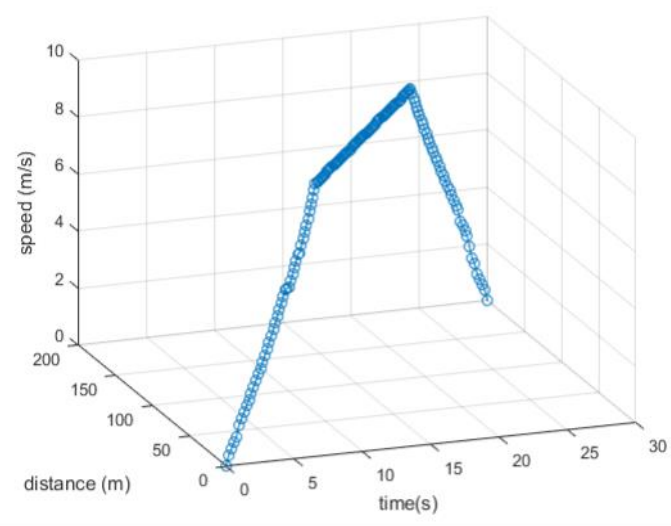

Fig. 4. Evolución del estado $[\mathrm{t}, \mathrm{x}, \mathrm{v}]$ en el tramo analizado

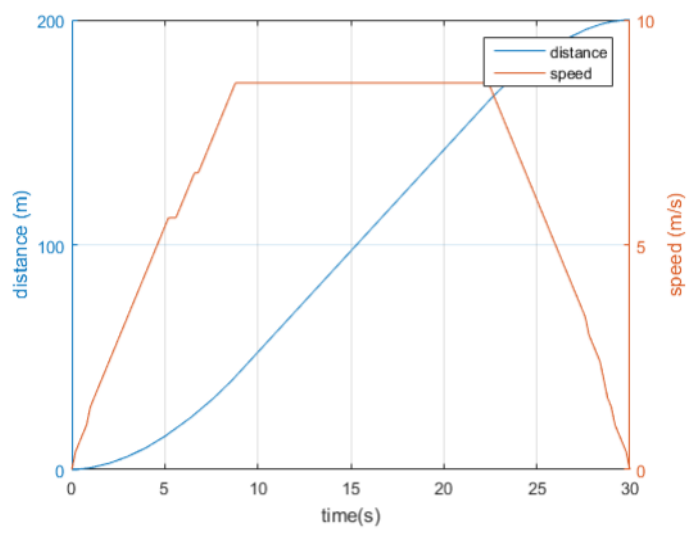

Fig. 5. Distancia recorrida y perfil de velocidad

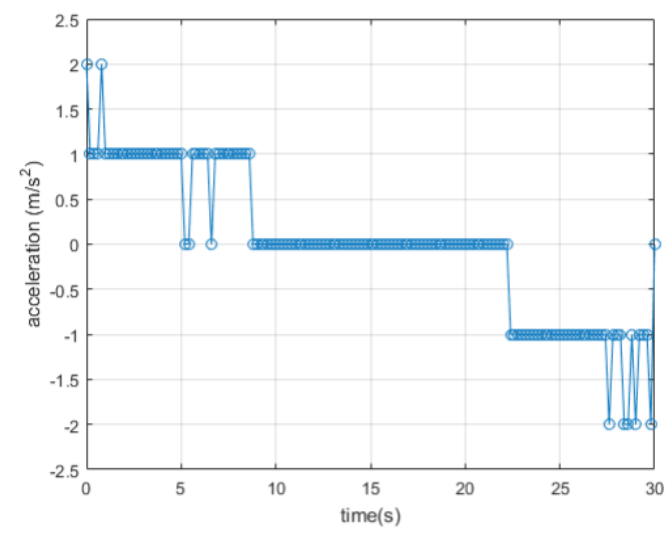

Fig. 6. Perfil de aceleración 
VI. CASO DE ESTUDIO: COMPARACIÓN CON RUTA REAL

Se ha seleccionado aleatoriamente una ruta para hacer la comparación con el trayecto óptimo que optimizaría el consumo de combustible. La ruta tiene una longitud de $11.1 \mathrm{Km}$ y está compuesta de un total de 34 ciclos de eco-driving debidos a las paradas del servicio, semáforos, stops y otras posibles detenciones provocadas por las características del tráfico. En la Fig. 7 se indican los perfiles de distancia y velocidad para los ciclos de eco-driving en la ruta seleccionada.

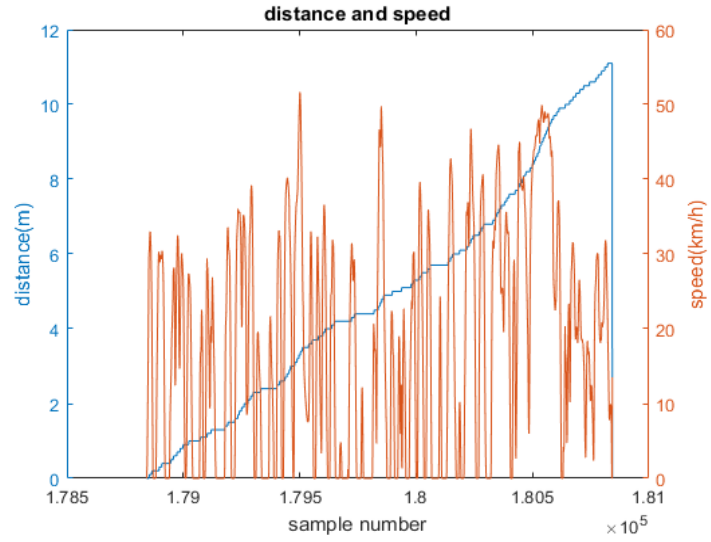

Fig. 7. Perfiles de distancia y velocidad en la ruta seleccionada

Se han identificado los ciclos de eco-driving a partir del perfil de velocidad y se ha ejecutado el algoritmo de optimización para cada uno de ellos. La duración de los ciclos y la distancia de cada uno de ellos puede apreciarse en la Fig. 8. Los ciclos tienen una duración media de 50,33 segundos y la distancia media es de 336,36 metros.

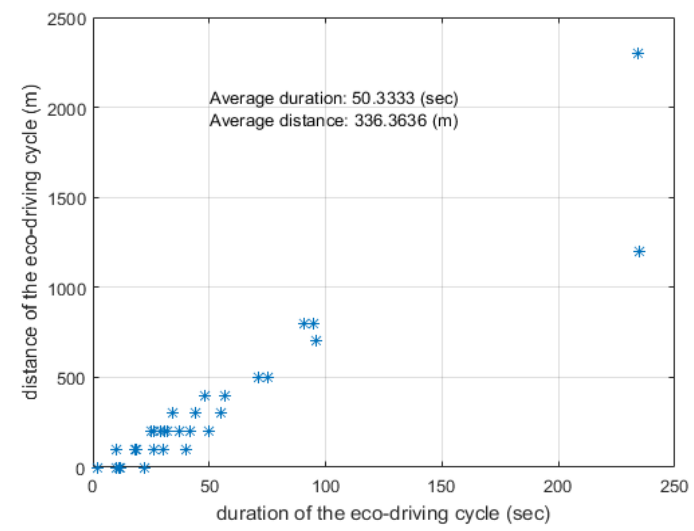

Fig. 8. Duración y distancia de los ciclos de eco-driving

En las figuras Fig. 9-12 se muestra una comparación entre los datos reales y los resultados de la simulación para los perfiles de velocidad y aceleración. Puede apreciarse una menor variabilidad en los perfiles de conducción óptima lo cual es indicador, evidentemente, de una conducción más moderada, tratando de mantener una velocidad constante una vez alcanzado el máximo en el ciclo correspondiente.

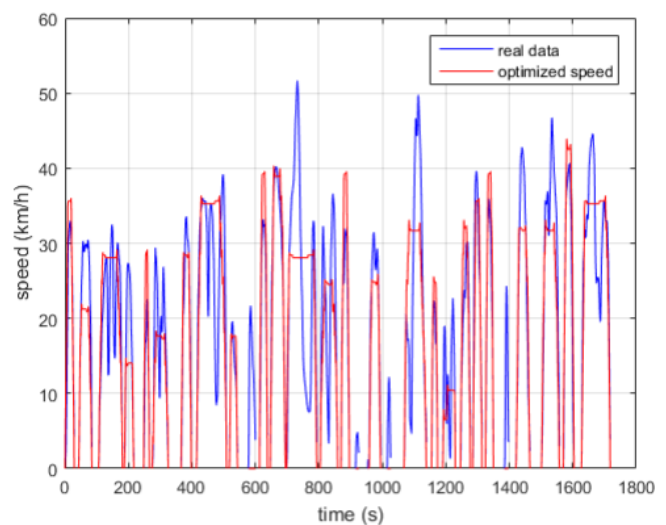

Fig. 9. Comparación de velocidades de ruta real y optimizada

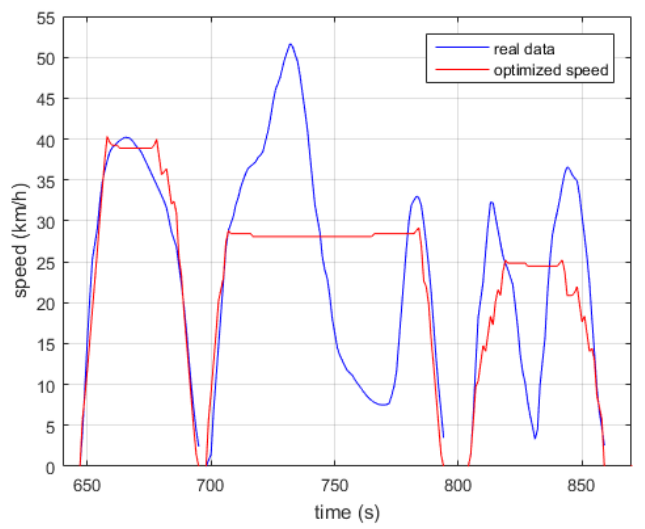

Fig. 10.Ampliación de velocidad en los ciclos de eco-driving \#11,12,13

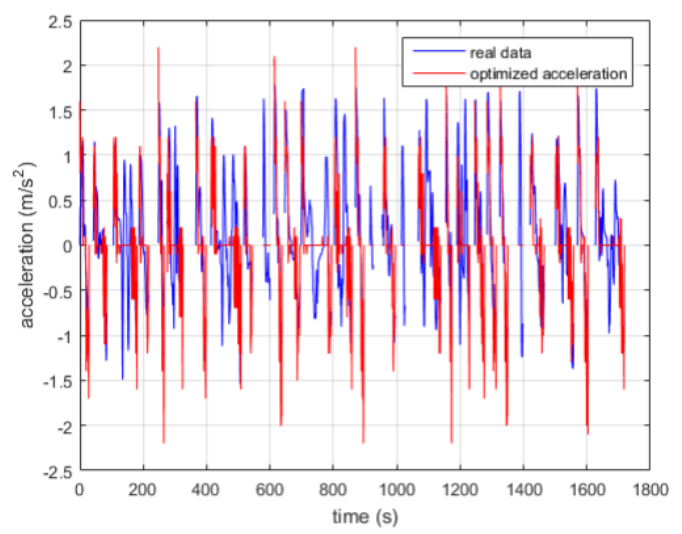

Fig. 11.Comparación de aceleraciones de ruta real y optimizada 


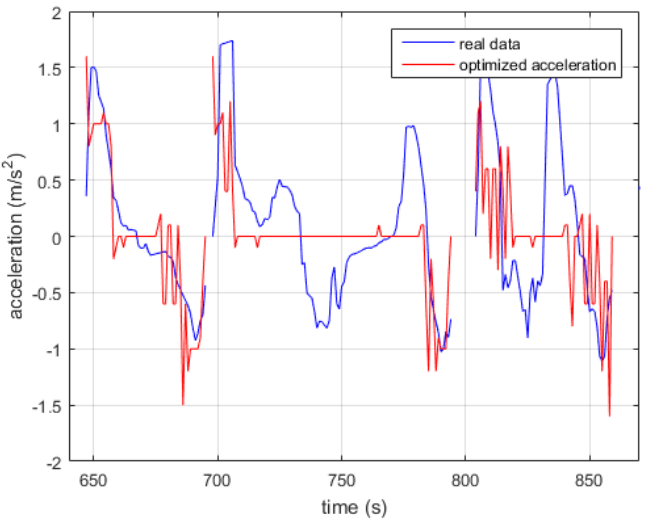

Fig. 12.Ampliación de aceleración en los ciclos de eco-driving $\# 11,12,13$

En el total de la ruta de $11.1 \mathrm{Km}$, el consumo real ha sido de 5,78 litros, con un promedio de 52,14 litros $/ 100 \mathrm{~km}$. Con la ruta optimizada el consumo total habría sido de 4,13 litros, con un promedio de 37,22 litros/100km. Estos datos indican que se podría haber reducido el consumo de combustible el $28,61 \%$. Ha de tenerse en cuenta que los resultados de la simulación se dan en condiciones óptimas de conducción, sin tráfico ni otros condicionantes externos como las condiciones meteorólogicas, estado de la vía, carga del vehículo, etc.

Extrapolando los resultados a todo el mes, en el que el vehículo analizado completó 611 rutas, la compañía podrían haber ahorrado 1008,2 litros de combustible. Para el total de la flota, donde los diferentes conductores de la empresa de transporte recorren miles de kilómetros, el ahorro de combustible sería de gran importancia para la compañía ya que supondría una importante reducción de costes y, por supuesto, redundaría en una mayor calidad medioambiental en la ciudad en la que presta servicio. Estos resultados avalan el uso de técnicas de conducción eficiente que, sin requerir de importantes inversiones en nuevos vehículos y tecnología, pueden conseguir resultados notables y mejoras medioambientales mediante cursos y estrategias de formación que optimicen el uso de la flota existente.

Para completar la formación de los conductores y servir de soporte a gerentes de flotas y formadores en conducción eficiente se dispone de la herramienta $\mathrm{VAT} \mathrm{ED}^{3}$ en la que, entre otras funcionalidades, se muestran gráficamente los patrones de conducción efiente e ineficiente en los diferentes puntos de la ruta, como se muestra en la Fig. 13.

3 Visual Analytics Tool for Evaluation of Drivers, Copyright O-712017

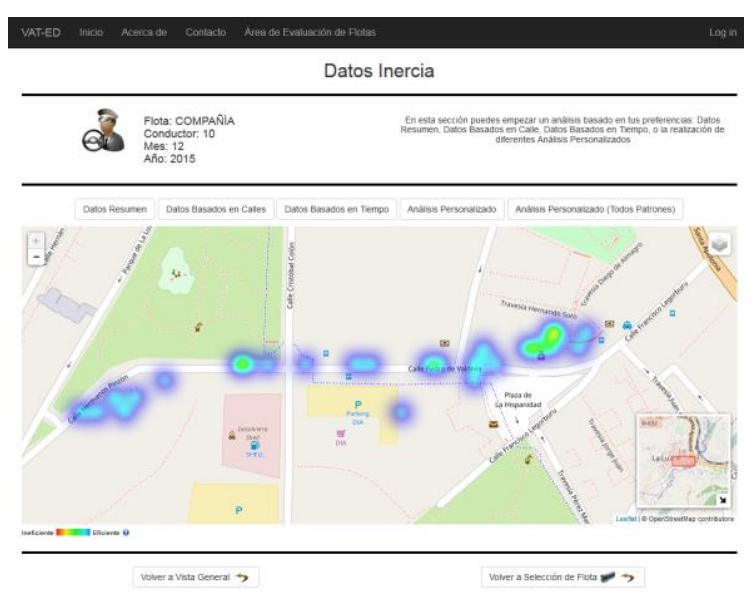

Fig. 13.Patrón de conducción en una zona del recorrido

Mediante esta herramienta gráfica de análisis, que permite seleccionar la flota, el conductor, la línea, el vehículo y el patrón de conducción se puede determinar la acción de conducción real ejecutada en un punto del recorrido. Complementando la información con los resultados de optimización mostrados en este artículo se potenciarían las técnicas de formación y con ello mejorarían los resultados en conducción eficiente de la compañía.

\section{CONCLUSIONES}

En este artículo se ha demostrado que la conducción eficiente es una alternativa válida para reducir el consumo de combustible $\mathrm{y}$, por consiguiente, la emisión de partículas nocivas al medioambiente. Esta reducción se puede lograr sin necesidad de realizar fuertes inversiones para renovar y/o mejorar la flota de vehículos existente.

El estudio realizado se ha centrado en el conductor y en él se pretende reforzar la importancia de una buena formación y motivación de los conductores para adoptar técnicas de conducción eficiente. Los algoritmos utilizados en este trabajo permiten determinar la acción óptima de conducción, de manera que se minimiza el consumo en una ruta determinada. Los resultados son de aplicabilidad directa en las estrategias de formación de conductores en conducción eficiente, ya que completan las ya existentes proporcionando una referencia para comparar la acción de conducción real con la óptima para esa ruta y vehículo. Asimismo, los resultados de este trabajo permiten disponer de un umbral óptimo de referencia para evaluar objetivamente las prestaciones relacionadas con la eficiencia de un determinado conductor. Otro campo en el que puede resultar interesante el estudio es el de la conducción automática. Así, los futuros vehículos sin conductor dispondrían de un patrón de aceleración/deceleración que optimizaría el perfil de velocidad haciendo mínimo el consumo en el trayecto a cubrir. 
Con los análisis realizados se ha demostrado que el ahorro de combustible puede alcanzar el $28 \%$, cifra significativa si se tienen en cuenta las grandes distancias recorridas por todos los conductores de la compañía a lo largo del año. El vehículo analizado completó 611 rutas a lo largo del mes con lo que, extrapolando los resultados obtenidos, la compañía podría haber ahorrado 1008,2 litros de combustible. No solo es importante el ahorro de combustible, sino que también esa reducción redunda en una atmósfera menos contaminada y en una mejor calidad de vida de la población donde se presta el servicio.

Por otro lado, ha de tenerse en cuenta que los resultados de la simulación se dan en condiciones óptimas de conducción, sin tráfico ni otros condicionantes externos como las condiciones meteorólogicas, estado de la vía, carga del vehículo, etc que tienen influencia en el consumo y no se han considerado en esta investigación. No es fácil disponer de toda esta información ya que depende de diferentes organismos e incluso el propio vehículo no dispone en ocasiones de los sensores necesarios. Los trabajos futuros irán encaminados a mejorar el modelo de consumo incluyendo todos estos factores.

Aunque el vehículo analizado es de cambio automático, se está trabajando en la mejora del modelo de consumo incluyendo las marchas engranadas y umbrales de cambio, de manera que se obtenga un modelo para cada marcha, probablemente mucho más preciso que el genérico presentado en este trabajo.

\section{AGRADECIMIENTOS}

Este trabajo ha sido financiado por la Comisión Europea (proyecto GlobalBLED SMEInst-10-20162017), el Plan Nacional de Investigación (proyecto MINECO-13-TIN2013-41749-R) y el Plan PCTI del Principado de Asturias (proyecto GRUPIN-14-065).

\section{REFERENCIAS}

[1] M. Di Natale, H. Zeng, P. Giusto, y A. Ghosal, Understanding and Using the Controller Area Network Communication Protocol. New York, NY: Springer New York, 2012.

[2] M. Rutty, L. Matthews, J. Andrey, y T. D. Matto, «Eco-driver training within the City of Calgary's municipal fleet: Monitoring the impact», Transp. Res. Part Transp. Environ., vol. 24, pp. 44 51 , oct. 2013 .

[3] M. Zarkadoula, G. Zoidis, y E. Tritopoulou, «Training urban bus drivers to promote smart driving: A note on a Greek ecodriving pilot program», Transp. Res. Part Transp. Environ., vol. 12, n. ${ }^{\circ} 6$, pp. 449-451, ago. 2007.

[4] H. K. Strömberg y I. C. M. Karlsson, «Comparative effects of eco-driving initiatives aimed at urban bus drivers - Results from a field trial», Transp. Res. Part Transp. Environ., vol. 22, pp. 28-33, jul. 2013.

[5] C. Vagg, C. J. Brace, D. Hari, S. Akehurst, J. Poxon, y L. Ash «Development and Field Trial of a Driver Assistance System to Encourage Eco-Driving in Light Commercial Vehicle Fleets», IEEE Trans. Intell. Transp. Syst., vol. 14, n. ${ }^{\circ} 2$, pp. 796-805, jun. 2013.
[6] J. C. Ferreira, J. de Almeida, y A. R. da Silva, «The Impact of Driving Styles on Fuel Consumption: A Data-Warehouse-andData-Mining-Based Discovery Process», IEEE Trans. Intell. Transp. Syst., vol. 16, n. ${ }^{\circ}$ 5, pp. 2653-2662, oct. 2015.

[7] A. Rionda et al., «Blended learning system for efficient professional driving», Comput. Educ., vol. 78, pp. 124-139, sep. 2014.

[8] O. F. Delgado, N. N. Clark, y G. J. Thompson, «Modeling Transit Bus Fuel Consumption on the Basis of Cycle Properties», J. Air Waste Manag. Assoc., vol. 61, n. ${ }^{\circ} 4$, pp. $443-$ 452, abr. 2011.

[9] E. Demir, T. Bektaş, y G. Laporte, «A comparative analysis of several vehicle emission models for road freight transportation», Transp. Res. Part Transp. Environ., vol. 16, n. ${ }^{\circ}$ 5, pp. 347-357, jul. 2011.

[10] Y. Saboohi y H. Farzaneh, «Model for developing an ecodriving strategy of a passenger vehicle based on the least fuel consumption», Appl. Energy, vol. 86, n. ${ }^{\circ}$ 10, pp. 1925-1932, oct. 2009.

[11] E. Hellström, J. Åslund, y L. Nielsen, «Design of an efficient algorithm for fuel-optimal look-ahead control», Control Eng. Pract., vol. 18, n. ${ }^{\circ}$ 11, pp. 1318-1327, nov. 2010.

[12] B. Saerens, J. Vandersteen, T. Persoons, J. Swevers, M. Diehl, y E. Van den Bulck, «Minimization of the fuel consumption of a gasoline engine using dynamic optimization», Appl. Energy, vol. 86, n. ${ }^{\circ}$ 9, pp. 1582-1588, sep. 2009.

[13] E. Velenis y P. Tsiotras, «Optimal Velocity Profile Generation for Given Acceleration Limits; The Half-Car Model Case», en Proceedings of the IEEE International Symposium on Industrial Electronics, 2005. ISIE 2005., 2005, vol. 1, pp. 361-366.

[14] F. Mensing, R. Trigui, y E. Bideaux, «Vehicle trajectory optimization for application in ECO-driving», en 2011 IEEE Vehicle Power and Propulsion Conference, 2011, pp. 1-6.

[15] A. Rionda Rodriguez, D. Martinez Alvarez, X. G. Paneda, D. Arbesu Carbajal, J. E. Jimenez, y F. Fernandez Linera, «Tutoring System for the Efficient Driving of Combustion Vehicles», IEEE Rev. Iberoam. De Tecnol. Aprendiz. RITA, vol. 8, n. ${ }^{\circ}$ 2, pp. 82-89, may 2013.

[16] A. Rionda etal., «UrVAMM \#x2014; A full service for environmental-urban and driving monitoring of professional fleets», en 2013 International Conference on New Concepts in Smart Cities: Fostering Public and Private Alliances (SmartMILE), 2013, pp. 1-6.

[17] A. G. Pañeda et al., «An Archictecture for a Learning Analytics System Applied to Efficient Driving», IEEE Rev. Iberoam. Tecnol. Aprendiz. RITA, vol. 11. n. ${ }^{\circ}$ 3, pp. 137-145, july 2016.

[18] K. Mokhtar y M. Z. Shah, «A regression model for vessel turnaround time», Tokyo Academic Industry \& Culture Integration Tour, pp. 10-19, 2006.

[19] D. E. Kirk, Optimal Control Theory: An Introduction. Mineola, N.Y: Dover Publications Inc., 2004. 